\title{
GROUPS WHOSE ORDERS ARE POWERS OF A PRIME*
}

\author{
BY \\ WILLIAM BENJAMIN FITE
}

In this article two somewhat distinct methods are used to study the properties of groups whose orders are powers of a prime. What may, for the purpose of distinction, be called the usual method is applied in the first part to establish certain properties of the commutators of a group. The commutators are differentiated among themselves by the introduction of the notion of successive commutators. The second part of the article concerns itself with the representation of certain groups as irreducible linear homogeneous groups and then by the use of the theory of group characters as developed by Frobenius and BurNSIDE establishes some properties of abstract groups.

\section{Part I.}

By a second commutator of a group $G$, of finite order, we mean a commutator formed by a commutator and any operator of $G$. Proceeding by successive steps, we define an $i$ th commutator as a commutator formed by an $(i-1)$ th commutator and any operator of the group. It follows from the definition that any $i$ th commutator is also a $j$ th commutator if $j<i$. In particular, every commutator is a first commutator. All the $i$ th commutators generate a subgroup which we shall call the ith commutator subgroup. $\dagger$ We shall represent it by $K_{i}$. It is evidently invariant in $G$.

If $G$ is of class $k$, identity is the only $k$ th commutator. Conversely, if identity is the only $k$ th commutator of $G$, then $G$ is of class $l$, where $l \leqq k$. Now in the quotient group $G / K_{i}$ identity is the only $i$ th commutator. This quotient group is therefore of class $l_{i} \leqq i$. Moreover if $L$ is any invariant subgroup of $G$ such that $G / L$ is of class $i$, then $L$ contains $K_{i}$. Hence if $l_{i}<i$, then $K_{i}$ coincides with $K_{i-1}$.

TheOREM I. A necessary and sufficient condition that a group $G$ have an

\footnotetext{
* Part I was presented to the Society September 16, 1904 ; part II, September 7, 1905. Received for publication March 18, 1905.

† This should not be confused with the $i$ th derived group.
} 
$\alpha, 1$ isomorphism with a group of class $i$ is that its $i$ th and $(i-1)$ th commutator subgroups be distinct.*

In order to make the foregoing statements apply to the case $i=1$, we agree that $K_{0}$ shall represent $G$ itself.

If $G$ has no invariant $i$ th commutators, except identity, the group $\dagger G^{(i)}$ has no invariant operators, except identity. Hence, if $i<k$, every group of class $k$ contains invariant $i$ th commutators besides identity. $\ddagger$

Theorem II. The operators of $G$ that correspond to the invariant operators of $G^{(i)}$ are commutative with all the ith commutators of $G$.

The theorem is true for $i=1 . \S$ We shall prove it true in general by induction. Let $t_{j}(j=1,2, \cdots)$ be any $j$ th commutator and let $s$ be any operator that corresponds to an invariant operator of $G^{(i)}$. We suppose that $s^{\prime}$ is commutative with $t_{i-1}^{\prime}$. Then $s^{-1} t_{i-1} s=t_{i-1} h, h$ being an invariant commutator of $G$. If $A$ is any operator of $G$, then $A^{-1} t_{i-1} A=t_{i-1} t_{i}$, and $s^{-1} A s=A t$, where $t$ is commutative with every $(i-1)$ th commutator, since it corresponds to an invariant operator of $G^{(i-1)}$. Hence

$$
\begin{aligned}
\left(s t^{-1}\right)^{-1} t_{i-1} t_{i}\left(s t^{-1}\right) & =t_{i-1} h \cdot s^{-1} t_{i} s \\
& =A^{-1} s^{-1} A \cdot A^{-1} t_{i-1} A \cdot A^{-1} s A=A^{-1} t_{i-1} h A=t_{i-1} t_{i} h .
\end{aligned}
$$

Therefore $s$ is communtative with $t_{i}$.

TheOREM III. If the ith commutator subgroup of a group $G$, of order $p^{m}$ ( $p$ a prime), is of order $p^{a}$, the class of $G$ is equal to, or less than, $\alpha+i . \|$

For identity is the only $i$ th commutator of $G^{(a)}$, and therefore $k^{(a)} \leqq i$. Hence $k \leqq \alpha+i$.

If $s_{j}\left(j=1,2, \ldots, k_{i}^{(i+1)}\right)$ be a set of any $k_{i}^{(i+1)}$ operators (not necessarily distinct) of $K_{i}$, and if $t_{1}$ is any operator of $K_{i}$, we have

$$
s_{j}^{-1} t_{j} s_{j}=t_{j} t_{j+1} \text {. }
$$

Now $t_{k_{i}^{(i+1)}}^{(i+1)}$ is invariant in $K_{i}^{(i+1)}$. Therefore $t_{k_{i}^{(i+1)}+1}$ is invariant in $K_{i}$ and $k_{i} \leqq k_{i}^{(i+1)}+1$.

Suppose now that $k \leqq 2 i+1$. Then $k^{(i+1)} \leqq i$, and in $G^{(i)}$ every ith commutator is invariant. Therefore $k_{i}=1$. Hence :

*Cf. Miller, Bulletin of the American Mathematical Society, vol. 4 (1898), p. 135.

† By $G^{(i)}$ we mean the $i$ th cogredient of $G$ (see DE SÉGuIER, Éléments de la théorie des groupes abstraits, p. 87); by $K_{j}^{(i)}$ we mean the $j$ th commutator subgroup of $G^{(i)}$.

‡Cf. MiLler, loc. cit., vol. 11 (1905), p. 367.

8 See Transactions of the American Mathematical Society, vol. 3 (1902), p. 351.

IISee Transactions of the American Mathematical Society, vol. 3 (1902), p. 350.

IThroughout this article we shall denote the class of $G$ by $k$, that of its $i$ th commutator sub. group by $k_{i}$, and that of its $j$ th cogredient group, $G^{(j)}$, by $k^{(j)}$. 
TheоRem IV. If a group $G$ is of order $p^{m}$ ( $p$ a prime), then $k_{i} \leqq k /(i+1)$,* $(i<k)$.

If $L_{2}$ is the second derived group of $G$, it is a divisor of $K_{3}$. This follows from the fact that $K_{1} / K_{3}$, being the commutator subgroup of $G / K_{3}$, which is of class 3 , is abelian.

Suppose $K_{i}$ is non-abelian. Then $k \geqq 2(i+1)$; and since the commutators that correspond to the invariant commutators of $G^{(i)}$ are invariant in $K_{i}$, the commutator subgroup of $G^{(j)}(j \leqq i+1)$ could not be cyclic.

If $K_{i}$ is of order $p^{a}, k \leqq \alpha+i$. Hence $k_{i} \leqq(\alpha+i) /(i+1)$. But since the operators of $G$ that correspond to operators of $G^{(i)}$ are commutative with all the operators of $K_{i}$, it follows that $K_{i}$ must either be abelian or contain at least $p^{2+1}$ invariant operators, if $G$ is of order $p^{m}$. Now if $k_{i}=(\alpha+i) /(i+1)$ and $a>1$, then the $x$ th cogredient group of $K_{i}$ would be of class 2 and order $p^{i+2}$ when $x=(\alpha+i) /(i+1)-2$. But this is impossible. We have therefore the

TheOREM V. If the ith commutator subgroup of a group of order $p^{m}(p$ a prime) is of order $p^{a}$, it is of class $k_{i}<(\alpha+i) /(i+1)$, except when $\alpha=1$, and then $k_{i}=(\alpha+i) /(i+1)=1$.

Suppose now that $G$, of order $p^{m}$, is non-abelian and contains two abelian subgroups, $G_{1}$ and $G_{2}$, of order $p^{m-1}$. Let $H$ denote the greatest common divisor of $G_{1}$ and $G_{2}$. It is of order $p^{m-2}$. Every operator of $H$ is invariant in $G$. Therefore $G^{\prime}$ is of order $p^{2}$ and $k=2$.

We proceed to show that if $G_{1}$ and $G_{2}$ are of class $k_{1}$, then $k \leqq \frac{1}{2}\left(k_{1}^{2}+k_{1}+2\right)$. We have just seen that this relation holds when $k_{1}=1$. We assume that it holds for all values of $k_{1}$ that are less than $i$. If $k_{1}=i$ and $k>i+1$, then every operator of $G$ all of whose commutators are invariant is in $H$. For if such an operator were not in $G_{1}$ ( or $G_{2}$ ) every invariant operator of $G_{1}$ (or $G_{2}$ ) would have all its commutators in $G$ invariant and $k^{\prime \prime} \leqq i-1$. Hence $k \leqq i+1$.

We suppose now that $k>i+1$. If every invariant operator of $G_{1}\left(\right.$ or $\left.G_{2}\right)$ is in $H$, its $(i-1)$ th commutators in $G$ must be invariant. Hence the subgroup of $G^{(i)}$ that corresponds to $G_{1}$ (or $G_{2}$ ) must be of class $l \leqq i-1$. If $G_{1}$ (or $G_{2}$ ) has an invariant operator that is not in $H$, while every invariant operator of $G_{2}$ (or $G_{1}$ ) is in $H_{0}$, then the subgroup of $G^{\prime}$ that corresponds to $G_{1}$ (or $G_{2}$ ) is of class $l \leqq i-1$. If both $G_{1}$ and $G_{2}$ have invariant operators not in $H$, every invariant operator of $H$ is invariant in $G$, and every invariant operator of $G$ is in $H$, since otherwise we should have $k=i$. Hence the subgroups of $G^{\prime}$ that correspond to $G_{1}$ and $G_{2}$ are of class $i-1$. In every case therefore $G^{(i)}$ has two subgroups of $1 / p$ th its order of classes equal to, or less than, $i-1$. By supposition then $k^{(i)} \leqq \frac{1}{2}\left\{(i-1)^{2}+(i-1)+2\right\}$. This gives $k \leqq \frac{1}{2}\left(i^{2}+i+2\right)$, and the proof by induction is complete.

* See Bulletin of the American Mathematical Society, vol. 9 (1902), p. 139. 
If $G_{2}$ is of class $k_{2}>k_{1}$, we proceed to show that $k \leqq \frac{1}{2}\left\{k_{1}\left(2 k_{2}-k_{1}\right)+k_{1}+2\right\}$. As we have just seen, this formula holds when $k_{2}=k_{1}$. We assume that the relation holds for all values of $k_{2}\left(\geqq k_{1}\right)$ up to, and including, $i-1$. Every invariant operator of $G_{2}$ is in $H$, since otherwise $G_{2}^{\prime}$ would be simply isomorphic with $H^{\prime}$ and therefore $k_{2}-1<k_{1}$. Hence every invariant operator of $G_{2}$ has all its $\left(k_{1}-1\right)$ th commutators in $G$ invariant. The subgroups of $G^{\left(k_{1}\right)}$ that correspond to $G_{1}$ and $G_{2}$ are therefore of classes $l_{1} \leqq k_{1}$ and $l_{2} \leqq i-1$ respectively. Hence, by our supposition,

$$
k^{\left(k_{1}\right)} \leqq \frac{k_{1}\left[2(i-1)-k_{1}\right]+k_{1}+2}{2}, \quad k \leqq \frac{k_{1}\left(2 i-k_{1}\right)+k_{1}+2}{2} .
$$

That is, if the formula holds for $k_{2}=i-1$, it holds for the next greater value of $k_{2}$. This result can be formulated into

Theorem VI. If a group $G$, of order $p^{m}$ ( $p$ a prime), contains two subgroups of order $p^{m-1}$ and classes $k_{1}$ and $k_{2}\left(\geqq k_{1}\right)$ respectively, then $k \leqq \frac{1}{2}\left\{k_{1}\left(2 k_{2}-1\right)+k_{1}+2\right\}$.

Let $G$ be of order $p^{m}$ and let $D$ be the greatest common divisor of all its invariant subgroups of index $p^{2}$. Then $D$ contains $K_{1}$ and $P_{2}$, the subgroup generated by the $p^{2}$ th powers of the operators of $G$. If $D$ is identity, then $G$ is abelian and has no operator of order greater than $p^{2}$. Conversely, if $G$ is abelian and has no operator of order greater than $p^{2}$, then $D$ is identity. Moreover, ${ }^{*} D \equiv\left\{K_{1}, P_{2}\right\}$.

If $D$ is the greatest common divisor of all the invariant subgroups of index $p^{\alpha}(\alpha>1)$ and is of order $p^{\gamma}$, then $k \leqq \alpha+\gamma-1$; for any such invariant subgroup gives a quotient group of class $l \leqq \alpha-1$, and hence $D$ contains the $(\alpha-1)$ th commutator subgroup.

\section{Part II.}

It is well known that the only substitutions of finite order permutable with every substitution of an irreducible linear homogeneous group of finite order are those which multiply each variable by the same number. Obviously then a necessary condition that a group be simply isomorphic with an irreducible groun is that its central be cyclic. $\dagger$ This condition is also sufficient if the group is the direct product of groups of orders $p_{1}^{m_{1}}, p_{2}^{m_{2}}, \cdots, p_{n}^{m_{n}}$ respectively $\left(p_{1}, p_{2}, \cdots, p_{n}\right.$ being distinct primes $)$. In proving this statement we shall use the following notation:

* The proofs of these statements are similar to those given by DE SÉGUIER, loc. cit., p. 115, for the subgroups of index $p$. Cf. BAgnera, Reale Accademia dei Lincei-A tti, Rendiconti, series 5, 1898, p. 63; Annali de Matematica, series 3, vol. 2 (1899), p. 264.

t The suhgroup formed by the invariant operators of a group is called the central of the group. This term seems to have been used for the first time by DE SÉGUIER, loc. cit., but he gives no explicit definition of it. 
$H$ is the cyclic central of $G$ of order $p_{1}^{\alpha_{1}} p_{2}^{\alpha_{2}} \cdots p_{n}^{a_{n}} ; H^{(i)}(i=1,2, \cdots, n)$ is the subgroup of $H$ of order $p_{i} ; G_{i}$ is the subgroup of $G$ of order $p_{i}^{m_{i}}$. The number of systems of conjugate operations of $G$ is $r$; the number of systems of conjugate operations of $G / H^{(i)} H^{(j)} H^{(i)} \ldots$ is $r_{i j k} \ldots$; the number of systems of conjugate operations of $G_{i}$ is $s_{i}$; the number of systems of conjugate operations of $G_{i} / H^{(i)}$ is $s_{i}^{\prime}$; the number of systems of conjugate operations of $G_{i} / H^{(i)}$ that (in $G / H^{(i)}$ ) correspond to operations of $G$ that give no invariant commutators besides identity is $t_{i}$.

If now $G$ were not simply isomorphic with an irreducible group, every irreducible representation of $G$ would also be a representation of at least one of the quotient groups $G / H^{(i)}$. The number of distinct irreducible representations of any group (of finite order) is equal to the number of its systems of conjugate operations.* If, therefore, we show that

$$
r>\sum_{k=1}^{n}(-1)^{k+1} r_{k} S_{n}
$$

where $r_{k s_{n}}$ denotes the sum of the ${ }_{k} C_{n} r$ 's with $k$ subscripts, it follows that the group under consideration is simply isomorphic with an irreducible group.

Now

$$
\begin{aligned}
r-r_{1} & =\prod_{i=2}^{n} s_{i} t_{1}\left(p_{1}-1\right), \\
r_{k} c_{n-1}-r_{k+1} c_{n}^{\prime} & =\prod_{i=2}^{n} s_{i} t_{1}\left(p_{1}-1\right) .
\end{aligned}
$$

The first subscript in the left member denotes a particular combination of the subscripts $2,3, \ldots, n$, while the second subscript is the same with the addition of 1 ; in the right member $\Pi^{\prime}$ indicates that in the product $s_{i}^{\prime}$ is to be placed for $s_{i}$ whenever $i$ occurs in both subscrips in the left member. If in equation. (II) we take all possible subscripts for a given $k$, and add, we get

$$
r_{k s_{n-1}}-r_{k+1} s_{n}^{\prime}=\sum \prod_{i=2}^{n \cdot \prime} s_{i} t_{1}\left(p_{1}-1\right) \text {. }
$$

From (I) and (III) we get

$$
r-\sum_{k=1}^{n}(-1)^{k+1} r_{k s_{n}}=\prod_{i=2}^{n}\left(s_{i}-s_{i}^{\prime}\right) t_{1}\left(p_{1}-1\right)=\prod_{i=1}^{n} t_{i}\left(p_{i}-1\right) .
$$

The right member of this equation is positive. Hence:

Theorem I. A necessary and sufficient condition that a group whose order is a power of a prime or a group which is the direct product of such

* The most direct proof of this is that given by BuRNside, Acta Mathematica, vol. 28 (1904), p. 36. Frobenius and BURNSIDE had given indirect proots before this.

Trans. Am. Math. Soc. 5 
groups be simply isomorphic with an irreducible linear homogeneous group is that its central be a cyclic group.

An irreducible abelian group operates upon only one variable. We consider now the number of variables operated upon by an irreducible non-abelian group. Let

$$
S: \quad x_{i}^{\prime}=\omega_{i} x_{i} \quad(i=1,2, \cdots, n),
$$

be the normal form of a non-invariant operation of an irreducible group $G$ that gives at least one invariant commutator besides identity, and suppose that

$$
\omega_{1}=\omega_{2}=\cdots=\omega_{\lambda_{1}} \neq \omega_{\lambda_{1}+1}=\omega_{\lambda_{1}+2}=\cdots=\omega_{\lambda_{2}} \neq \omega_{\lambda_{2}+1}=\cdots
$$

We select $R$ so that $S^{-1} R S=R T$, where $T$ is the invariant commutator of highest order given by $S$. Now $T$ is of the form

and therefore

$$
x_{i}^{\prime}=\omega x_{i} \quad(i=1,2, \cdots, n),
$$

if

$$
\omega_{i}^{-1} \sum_{t=1}^{n} b_{i t} \omega_{t} x_{t}=\sum_{s=1}^{n} b_{i s} \omega x_{s},
$$

$$
R: \quad x_{i}^{\prime}=\sum_{s=1}^{n} b_{i n} x_{s} \quad(i=1,2, \cdots, n) .
$$

If $\omega_{i}^{-1} \omega_{j} \neq \omega$, then $b_{i l}=0$ when $j$ and $l$ are both between $\lambda_{r-1}$ and $\lambda_{r}+1$. But if $\omega_{i}^{-1} \omega_{j}=\omega, b_{i l}$ is not necessarily zero. Now $\omega_{i}^{-1} \omega_{j}$ will still equal $\omega$ if $j$ takes any one of $\lambda_{r}-\lambda_{r-1}$ values, and $i$ takes any one of, say; $\lambda_{s}-\lambda_{s-1}$, values. Since the determinant of $R$ does not vanish, we must have $\lambda_{r}-\lambda_{r-1}=\lambda_{s}-\lambda_{s-1}$. If $\omega_{j}^{-1} \omega_{k}=\omega$, then $\lambda_{s}-\lambda_{s-1}=\lambda_{t}-\lambda_{t-1}$, and so on in a cycle until we get back to $\omega_{i}$. The number of elements in this cycle equals the order of $T$, and the sum of the $\omega_{i}$ 's composing the cycle is zero. Therefore

$$
\sum_{i=1}^{n} \omega_{i}=0 \text {. }
$$

If $S$ corresponds to an invariant operator of $G^{\prime}$, then $\lambda_{1} t=n$, where $t$ is the order of * $T$. have

* Since $R^{-1} S R=S T^{-1}$ and the characters of any two conjugate operations are the same, we

Therefore

$$
\sum_{i=1}^{n} \omega_{i}=\omega^{-1} \sum_{i=1}^{n} \omega_{i}
$$

$$
\sum_{i=1}^{n} \omega_{i}=0
$$

This is a much shorter proof than the one given above. The latter however has the advantage of showing also that the number of variables cannot be less than the number of invariant commutators. 
Let $N$ be the order of $G$ and $r$ the number of its systems of conjugate operations. If $\chi_{k}^{i}(k=1,2, \cdots, r)$ is the set of characters of $G$ corresponding to the irreducible representation $g_{i}$, and if $h_{k}$ is the number of operations in the $k$ th conjugate set, then *

$$
\sum_{k=1}^{r} h_{k} \chi_{k}^{i} \chi_{k}^{i \prime}=N
$$

If we take $g_{i}$ as $G$ itself, this can be put into the form

$$
h n^{2}+\sum^{\prime} h_{k} \chi_{k}^{i} \chi_{k}^{i^{\prime}}=N,
$$

where the summation extends over all the conjugate sets that are composed of more than one operation. But $\chi_{k}^{i}$ and $\chi_{k}^{i \prime}$ are conjugate imaginaries and therefore $\chi_{k}^{i} \chi_{k}^{i \prime}$ is real and positive. If every non-invariant operation of $G$ gives an invariant commutator besides identity, every term in this summation is zero. Hence :

Theorem II. The number of variables in an irreducible linear homogeneous group $G$ is equal to the square root of the order of $G^{\prime}$, if every noninvariant operation of $G$ gives an invariant commutator besides identity. The number of variables is less than this, but not less than the number of invariant commutators if $G$ contains an operation the sum of whose multipliers is not zero.

In particular, if $G$ is an irreducible metabelian group of order $N$ in $n$ variables, then $n=\sqrt{N / h}$, where $h$ is the order of the central of $G$.

Since the number of variables in an irreducible group of order $p^{m}$ is greater than, or equal to, the order of its $(k-1)$ th commutator subgroup, it follows that, if the central of $G$ is cyclic, the order of $G^{\prime}$ must be greater than, or equal to, $p^{2 \beta}$, where $p^{\beta}$ is the order of the $(k-1)$ th commutator subgroup. If $G^{\prime}$ is of order $p^{2 \beta}$, every non-invariant operation of the irreducible group that is simply isomorphic with $G$ must have the sum of its multipliers equal to zero.

TheOREM III. If the central, $H$, of a group $G$, of order $p^{m}(p$ an odd prime), is cyclic and of order $p^{h}$, then $m-h$ must be even when $K$ is cyclic. $\dagger$

For if $m-h$ is odd, $G$ must contain a non-invariant operation $R$ that gives no invariant commutator besides identity. Now $R$ must give some commutators besides identity. Suppose that it gives a commutator $S$ in the $(k-i)$ th commutator subgroup, but none in any higher commutator group ; say $R^{-1} A R=A S$. If $S^{p \gamma}$ is the first power of $S$ that is contained in the $(k-i+1)$ th commutator subgroup, then, provided $K$ is cyclic, $R^{-1} A^{p \gamma} R=A^{p \gamma} S_{1}$, where $S_{1}$ is contained

* Frobenius, Berliner Sitzungsberichte, 1896, II, p. 1359 ; Burnside, Proceedings of the London Mathematical Society, vol. 33 (1900), p. 154 ; series 2, vol. 1 (1903), p. 123.

† For a special case, see Transactions of the American Mathematical Society, vol. 3 (1902), p. 342. 
in the $(k-i+1)$ th commutator subgroup and is distinct from identity.* But this contradicts the hypothesis. The theorem is therefore proved.

Let $H_{i}$ be the $i$ th central $\dagger$ of $G$. We shall denote its order by $p^{h_{4}}$. If $K$ is cyclic, the subgroup of $G$ that corresponds to the invariant operations of $G^{(j)}$ of order $p^{2 a+1}$, or less ( $\alpha$ an integer), cannot be cyclic. If it were cyclic, we could denote by $\bar{H}_{j}$ the subgroup of $H_{j}$ of order $\ddagger p^{h_{j-1}+2 a+1}$. This would be invariant in $G$ and the commutator subgroup of $G / \bar{H}_{j}$ would be cyclic, as would also its central. But the order of its first cogredient would be $p^{m-h_{j}-2 a-1}$, which would be an odd power of $p$, since $p^{m-h_{j}}$, being the order of the first cogredient of $G / H_{j-1}$, would be an even power of $p$.

If the subgroup of $G$ that corresponds to the invariant operations of $G^{(j)}$ of order $p^{2 a}$, or less, were cyclic, then the subgroup corresponding to the invariant operations of $G^{(j)}$ of order $p^{2 a-1}$, or less, would be cyclic, and this has just been seen to be impossible. Hence if $K$ is cyclic, no central except the first can be cyclic.

Professor BuRnside has discussed irreducible groups in a prime number of variables with reference to their solubility.§ We shall here consider such groups, of order $p^{m}$, with reference to their classes.

Let $G$ be such a group. It is non-abelian and $K_{k-1}$ is of order $p$. If $k=2$, $G^{\prime}$ is of order $p^{2}$. If $k>2, G$ contains a commutator, $S$, that corresponds to an invariant commutator of $G^{\prime}$ of order $p$. Therefore $S$ has just $p$ conjugates and is invariant in a subgroup, $G_{1}$, of $G$ of order $p^{m-1}$. This subgroup is reducible and therefore abelian, and it contains $K$. Suppose that $A$ is so chosen that $G \equiv\left\{G_{1}, A\right\}$. Then $A^{p}$ is invariant in $G$ and $A$ has $p^{m-a-1}$ conjugates. The order of $K$ is therefore at least $p^{m-a-1}$. If the order of $K$ were greater than this the commutator subgroup of $G^{\prime}$ would be of order greater than $p^{m-a-2}$. But this is impossible. Therefore the order of $K$ is $p^{m-a-1}$. The order of $K_{2}$ is $p^{m-a-2}$ and, in general, the order of $K_{i}(i=1,2, \ldots, k)$ is $p^{m-a-i}$. Hence $k=m-\alpha$, and the $p^{r}$ th power of every operator of $G$ is invariant if $\| r(p-1) \geqq m-\alpha-1$.

CornelL Universtty, March, 1905.

* If $p=2, S_{1}$ may be identity. This is the case in the groups of order $2^{4}$ and class 3 . The theorem is not true in the case of these groups.

† See de Sḱquirr, 100. cit., p. 87.

$\ddagger$ In order that this may be applicable when $j=1$, we agree that $h_{0}$ shall be zero.

Lota Mathematioa, vol. 27 (1903), p. 217.

|l Bulletin of the American Mathematical Society, vol. 10 (1904) p. 347. 